\title{
Piffalls in scoring MR images of rheumatoid arthritis wrist and metacarpophalangeal joints
}

\author{
F McQueen, M Østergaard, C Peterfy, M Lassere, B Ejbjerg, P Bird, P O'Connor, H Genant, \\ R Shnier, P Emery, J Edmonds, P Conaghan
}

Ann Rheum Dis 2005;64(Suppl I):i48-i55. doi: 10.1136/ard.2004.031831

This paper outlines the most important piffalls which are likely to be encountered in the assessment of magnetic resonance images of the wrist and metacarpophalangeal joints in patients with rheumatoid arthritis. Imaging artefacts and how these can be recognised using various sequences and views are discussed. Normal structures such as interosseous ligaments and nutrient foramina may appear prominent on certain images and need to be identified correctly. Pathological change in the rheumatoid hand involves many tissues and when substantial damage has occurred, it may be difficult to identify individual structures correctly. Bone erosion, bone oedema, synovitis, and tenosynovitis frequently occur together and in close proximity to each other, potentially leading to false positive scoring of any of these. Examples are given to illustrate the various dilemmas the user of this atlas may face when scoring the rheumatoid hand and suggestions are made to assist correct interpretation of what can be very complex images.

See end of article for authors' affiliations

Correspondence to: Prof $M$ Østergaard, Department of Rheumatology, Copenhagen Úniversity Hospital at Hvidovre, Kettegaard alle 30, DK2650 Hvidovre, Denmark; mo@dadlnet.dk
B ecause magnetic resonance imaging (MRI) is such a sensitive technique for imaging soft tissue inflammation and bony change in rheumatoid arthritis (RA), it is possible for the reader to misinterpret various features and potentially score false positives or false negatives using the Outcome Measures in Rheumatology Clinical Trials (OMERACT) RA MRI scoring (RAMRIS) system. ${ }^{1}$ There are some artefacts related to the imaging process itself that may confuse interpretation. There are also many sites of potential pathology in the rheumatoid hand and inflammation of, or structural damage to, some structures may mimic involvement of others. These are possible pitfalls for the user of the EULAR-OMERACT RA magnetic resonance imaging reference image atlas and some of the commoner ones are outlined in this paper, organised as follows:

\footnotetext{
- Artefacts

- Normal features that could be misinterpreted

- Influence of slice thickness/field of view

- Distortion of anatomy due to disease
}

- Bone erosions and bone oedema

- Synovitis and tenosynovitis

\begin{abstract}
ARTEFACTS
Like any other imaging modality, MRI is susceptible to artefacts. However, fortunately, MR scans can be performed in any plane and use several pulse sequences which allow the majority of artefacts to be identified as such. The following are examples of some artefacts which may cause problems when interpreting images from patients with RA.
\end{abstract}

\section{Susceptibility artefacts}

Adjacent tissues with different "susceptibilities" (that is, degree to which they become magnetised by the external magnetic field) produce fluctuations in their local magnetic field. If these field variations are significant, a wide range of precessional frequencies (associated with recovery of spin of hydrogen nuclei after withdrawal of the external radio frequency pulse) appear within a voxel and the signal can be greatly decreased as they cancel out each other. Susceptibility artefacts occur most commonly at interfaces between trabecular bone and soft tissue or between air and soft tissue.

\section{Metal artefact}

This is an example of a severe susceptibility artefact. The presence of any metal in the region being imaged will severely distort the magnetic field and alter the image. ${ }^{2}$ An example is shown in fig 1 where a metal pin across the distal radioulnar joint has resulted in blurring and signal irregularity affecting the rest of the image (which nevertheless shows evidence of erosive damage in this patient with aggressive RA).

\section{Partial volume artefacts}

These occur when a structure is only partly contained within the imaging section, voxel or pixel, and are most marked in regions where two tissues of different signal intensity adjoin such as at the interface between inflamed synovial membrane and bone. In one plane this may lead to blurring of a bony border, simulating erosion,

\footnotetext{
Abbreviations: EULAR, European League Against Rheumatism; FOV, field of view; MCP, metacarpophalangeal; $M R I$, magnetic resonance imaging; OMERACT, Outcome Measures in Rheumatology Clinical Trials; RA, rheumatoid arthritis
} 


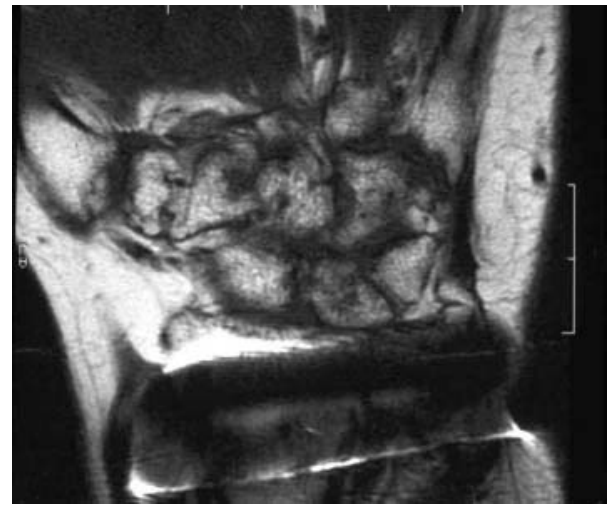

Figure $1 \mathrm{~T} 1$ weighted ( $\mathrm{T} 1 \mathrm{w}$ ) coronal image of the wrist in a patient with $R A$ in whom a metal screw was used for surgical fixation of the distal radioulnar joint.

but examination of the image in another plane will often show bony borders to be clearly defined (fig 2).

\section{Chemical shift artefact}

This is another artefact, which may occur where two tissues of very different composition are adjacent to one another, such as fat next to water (or synovial fluid). Different
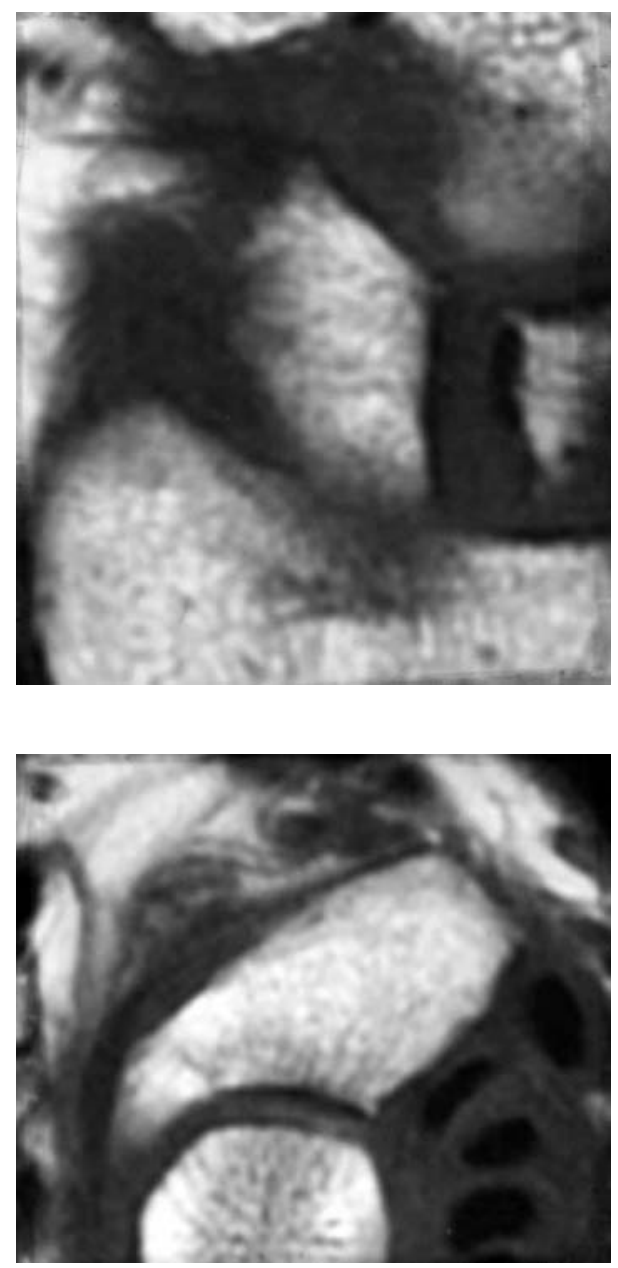

Figure 2 (A) T1 weighted coronal image of the scaphoid where the radial border is blurred because of adjacent synovial membrane with lower signal, "averaging" with high signal of bone marrow. (B) T1 weighted axial image showing the scaphoid margin is not eroded.

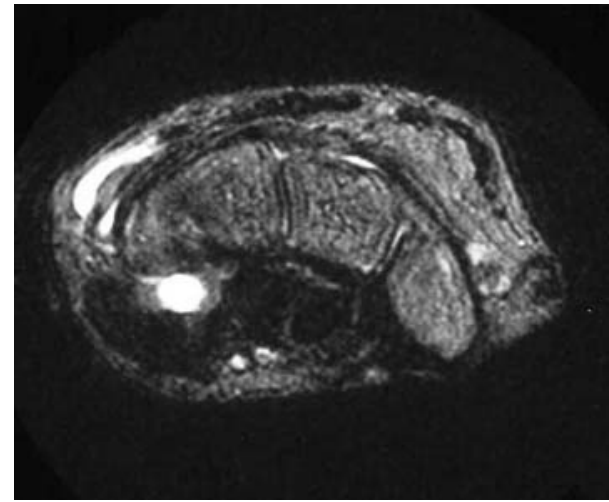

Figure 3 T2 weighted fat saturated (T2w FS) axial image of the wrist showing movement artefact.

molecular environments influence the protons within these tissues causing differences in their Larmor (resonant) frequencies. This effect is directly proportional to magnetic field strength and so is more pronounced when the external magnetic field is of relatively high strength, such as $1.5 \mathrm{~T}$. The result is that in these regions, there are shifts in frequency of the two differing tissues along the read-out gradient causing pixel displacement. On the MR image, this may result in adjacent lines of hyperintensity and hypointensity at the tissue interface. Echo planar imaging is particularly sensitive to chemical shift artefacts but these can be minimised with fat saturation techniques. ${ }^{3}$

\section{Truncation artefact}

The signal emitted by protons in the tissue being scanned can be described mathematically as a series of sinusoid curves. The spatial frequency of these sinusoids dictates the clarity of the image. However, in places where there are anatomical edges to be defined (such as the edge of the patellar cartilage), there are sharp changes in signal intensity, and these can produce artefactual ripples. Because these ripples result from truncating the infinite series of sinusoids that would be necessary to produce a sharp edge on the image, they are referred to as truncation artefacts. ${ }^{4}$

\section{Wraparound artefact}

This artefact is produced when the dimension of the area being imaged (such as the wrist) is larger than the field of view (FOV) in this direction. Structures from outside the FOV are then shown on the opposite side of the image. ${ }^{3}$

\section{Movement artefact}

Like any imaging modality, MR image clarity is significantly affected by movement. This is more of a problem than with plain radiographs as the patient having an MR scan is required to remain absolutely still for a much longer period. They may also be in an uncomfortable position (prone with arm above head or "superman position" to image the wrist in some scanners), which is particularly difficult to sustain for those with inflammatory arthritis of the shoulder. ${ }^{4}$ T2 weighted sequences require the patient to stay still for the longest periods and are therefore especially prone to movement artefact (fig 3).

\section{Pulsation artefact}

Because blood velocity changes through the cardiac cycle, images with total scan times much longer than the cardiac cycle may reflect fluctuations in data recovery leading to extra shadows near pulsatile vessels. This will happen only on the phase encoding axis as frequency encoding is very fast relative to pulsations. This artefact is usually recognisable 


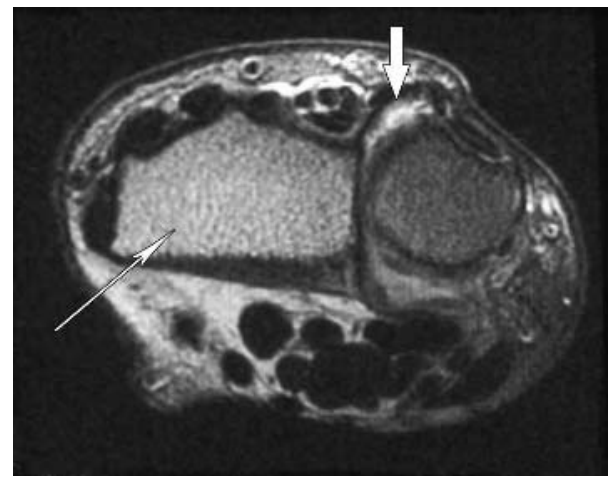

Figure 4 T2 weighted axial image of the distal radius and ulna. Fat saturation is complete for the ulna but not for the radius, where "shine through" of marrow fat is apparent (thin arrow). This appearance mimics bone oedema. Fat in subcutaneous tissues is also bright in the left half of the picture but not in right half where bright signal in the distal radioulnar joint indicates synovitis (wide arrow).

because it is the same size as the vessel and spreads right across the image in the phase encoding direction in the same manner as a movement artefact. ${ }^{3}$

\section{Inhomogeneity of fat suppression}

This problem occurs when the area being imaged lies within a part of the magnetic field that is not completely uniform. This might occur during imaging of the wrist if the patient is lying in the magnet with their arm by their side-meaning that this region is at the edge of the magnetic field. ${ }^{4}$ Fat suppression can become inhomogeneous and result in an appearance mimicking bone oedema ${ }^{5}$ as in fig 4 .

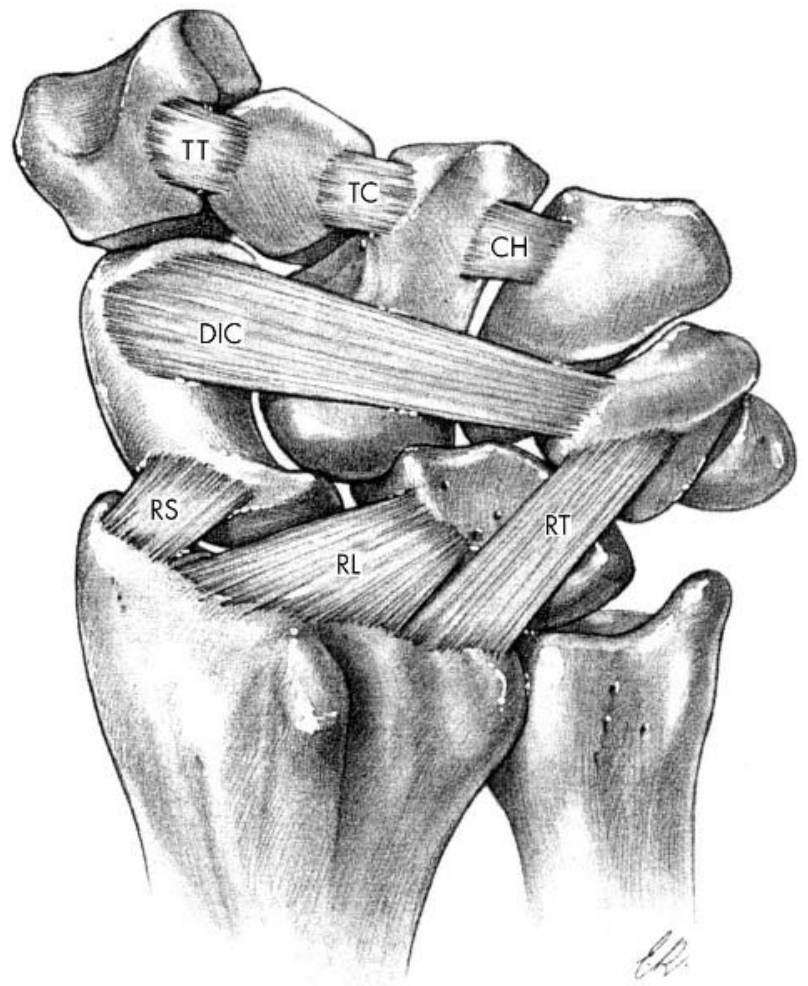

Figure 5 Palmar interosseous ligaments of the wrist (Reprinted from Taleisnik, J. The Wrist, Figure 2-18, copyright 1985, with permission from Elsevier Inc.).

\section{NORMAL FEATURES THAT COULD BE MISINTERPRETED}

A thorough review of the anatomical variants of the hand and wrist, as seen on MRI, is beyond the scope of this paper. We include here only those normal appearances that may cause confusion in interpreting MR images from the patient with RA.
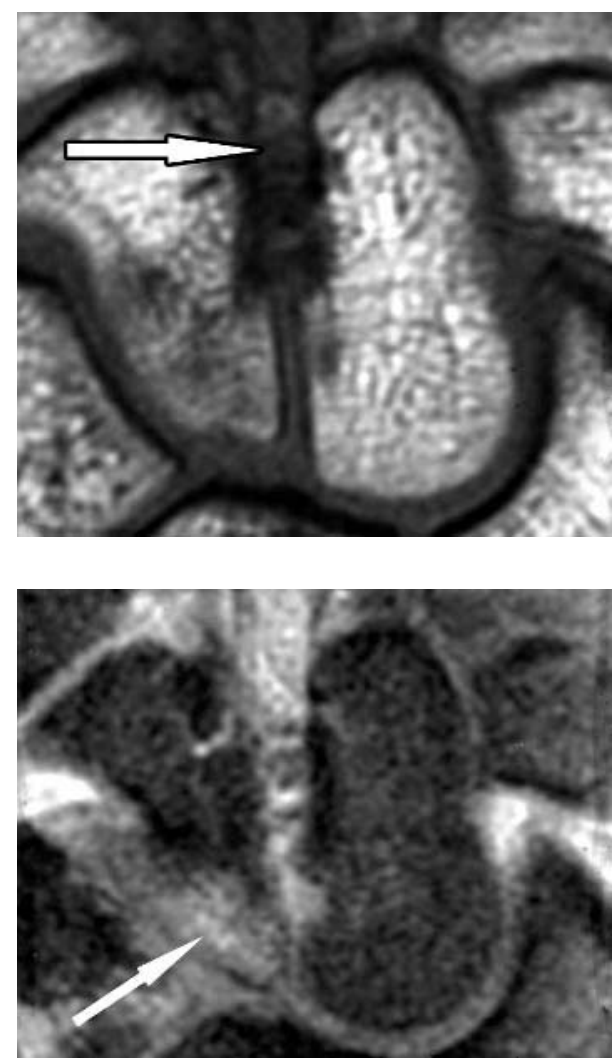

Figure 6 (A) T1 weighted coronal image of the wrist from a patient with early RA. The interosseous ligament between the capitate and hamate blurs the inner borders of these bones distally (arrow). This appearance can mimic erosion (but erosion may also occur adjacent to this ligament). (B) On the T2 weighted fat saturated image, the ligament and adjacent synovial membrane are bright suggesting inflammation. There is also bright signal (arrow) in the hamate revealing bone oedema (seen as grey on T1 weighted image).

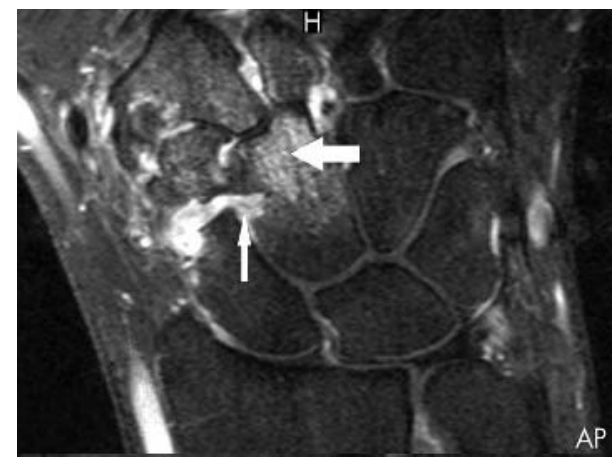

Figure 7 Short tau inversion recovery (STIR) coronal image of the wrist reveals an interosseous ligament between the capitate and scaphoid (thin arrow). There is also bone oedema within the distal capitate (wide arrow). 


\section{Interosseous ligaments at the wrist}

The intrinsic interosseous ligaments of the wrist serve to stabilise the carpus and are present on the palmar and dorsal surfaces. The palmar ligaments (fig 5) are usually thicker and insert into multiple carpal bones and the major dorsal ligament is the radioscaphoid ligament. On certain MR sequences, interosseous ligamentous attachments can simulate erosions and need to be distinguished from these. Examples are shown in figs 6 and 7.

\section{Articular ligaments attaching to metacarpophalangeal (MCP) joints}

Each MCP joint has one palmar and two collateral ligaments (fig 8). The collateral ligaments insert into bony recesses at the metacarpal heads. In RA where there is florid MCP joint synovitis, there may be inflamed synovial membrane adjacent to these bony recesses, simulating erosion. These areas of bone adjacent to ligamentous attachments are also susceptible to early erosion, so distinguishing between normal and pathological appearances can be difficult.

\section{Lesions simulating erosions}

Ejbjerg et al have recently shown that small erosion-like lesions occur in around $2 \%$ of metacarpal and wrist bones in normal subjects. ${ }^{6}$ These lesions (which most often occurred in the capitate and lunate) did not tend to enhance post contrast, as is typical of active rheumatoid erosions. Another point of difference in Ejbjerg's study was that bone oedema was not seen in normal subjects.

\section{Nutrient foramina}

In the bones of the carpus, nutrient foramina (especially prominent in the lunate) may be apparent on certain sequences and might be mistaken for small erosions (fig 9).

\section{INFLUENCE OF SLICE THICKNESS/FIELD OF VIEW}

The optimal slice thickness and field of view for imaging the rheumatoid hand are important considerations when documenting damage progression. A slice thickness of $3 \mathrm{~mm}$ or less with an inplane resolution of at least $1 \mathrm{~mm}^{2}$ is usually required to detect fine anatomical detail. However, even with a slice thickness of $3 \mathrm{~mm}$, small erosions may be missed in one plane, due to partial volume averaging. If the definition of erosions requires visualisation in two planes, as it does for
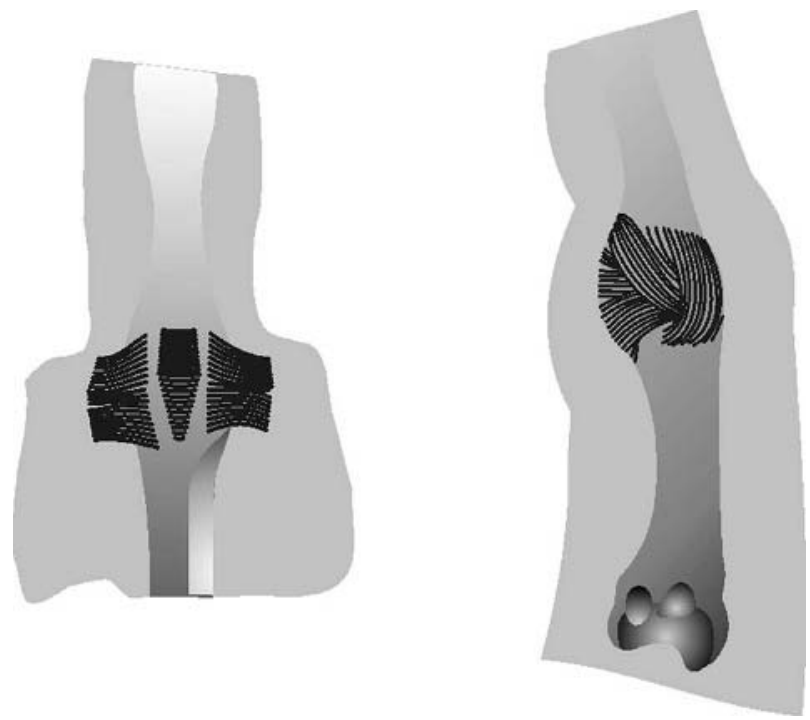

Figure 8 Interosseous ligaments as they attach to the metacarpal head and the base of the proximal phalanx.

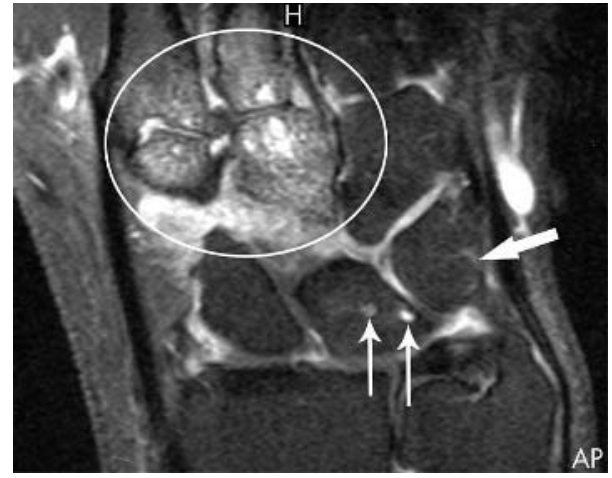

Figure 9 Short tau inversion recovery (STIR) coronal image of the carpus showing a nutrient vessel within the triquetrum and two nutrient foramina within the lunate mimicking erosions (thin arrows). True erosions with surrounding bone oedema are shown within the distal capitate and trapezoid bones and adjacent metacarpal bases (circle).

the OMERACT RA MRI scoring (RAMRIS) system, ${ }^{1}$ there will be some lesions that do not fulfil these criteria as they have simply been missed due to positioning of the slices. This imposes a "floor" effect for small lesions, which is higher in size terms than would be expected from the "within slice" resolution. Thinner slices can be used and evidence suggests more detail can be seen but the image analysis time is
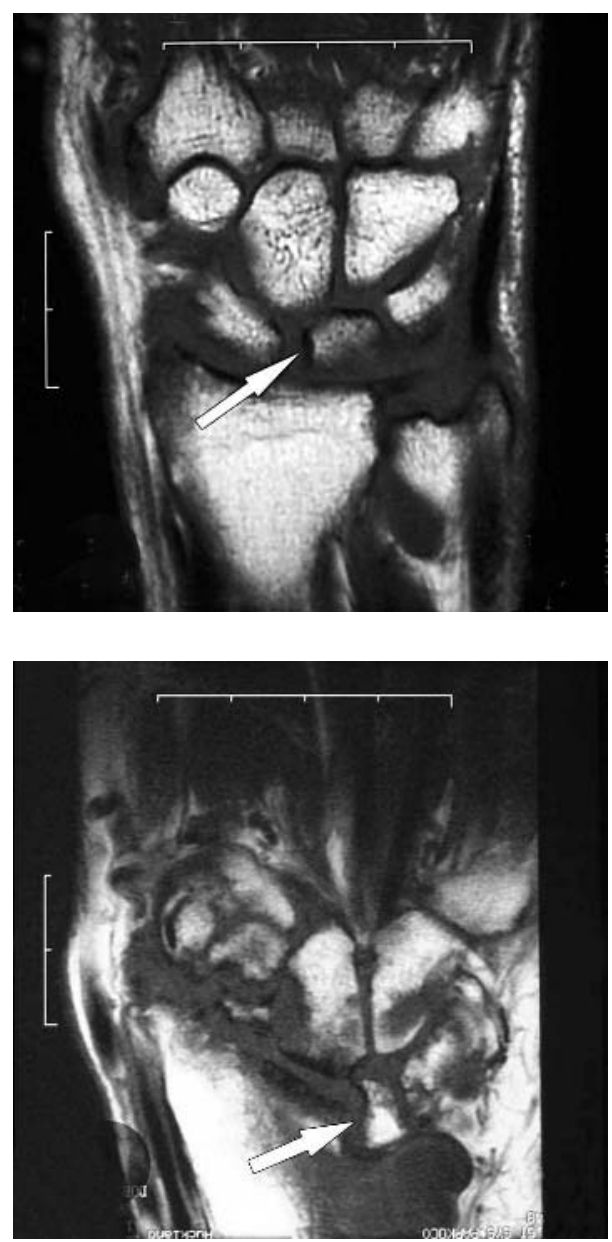

Figure 10 (A) $\mathrm{Tl}$ weighted coronal image of the wrist from a patient with early RA (six months from onset). (B) T1 weighted coronal image of the same region six years later showing gross deformity of the joint with erosion and distal migration of the lunate (arrow). 

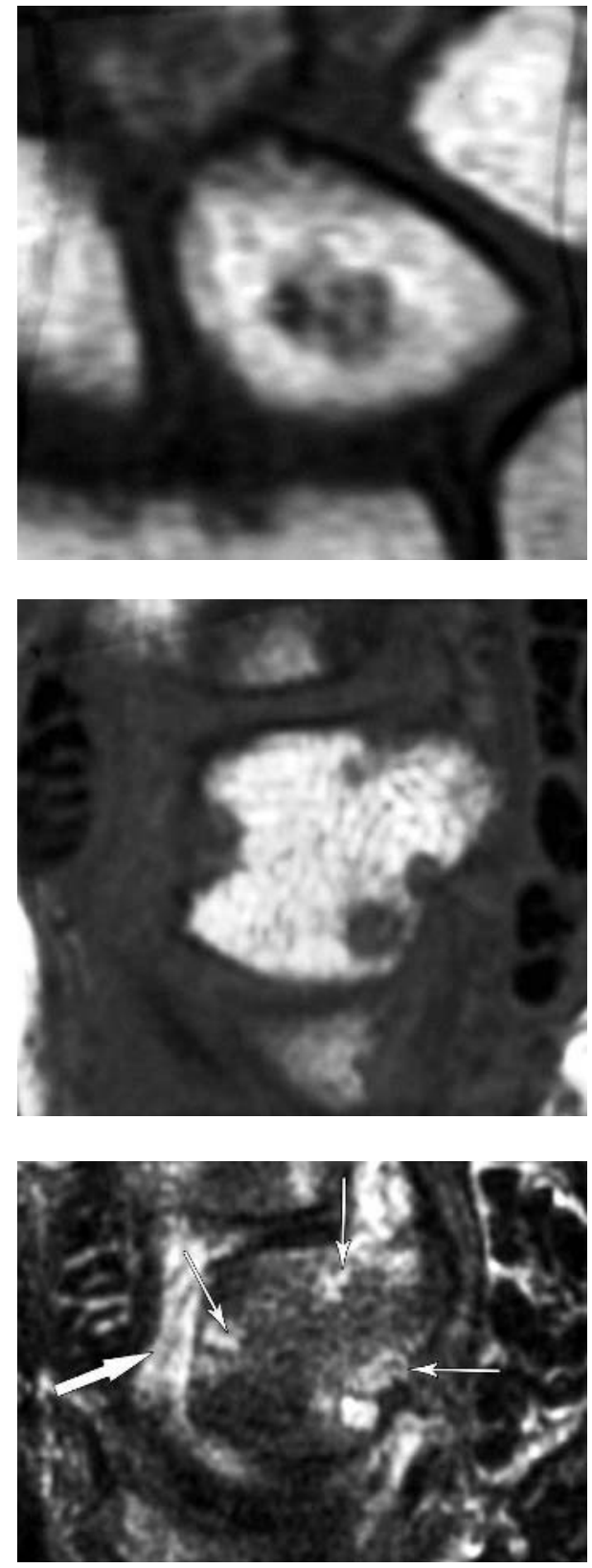

Figure 11 (A) $\mathrm{Tl}$ weighted coronal image of the wrist. The central lesion in the lunate might be localised bone oedema or erosion - an axial view is required. (B) T1 weighted axial image reveals several erosions. (C) T2 weighted fat saturation axial image showing bone oedema surrounding erosions (thin arrows) and adjacent synovitis (thick arrow).

dramatically increased. ${ }^{7}$ The signal to noise (SNR), defined as the ratio of the amplitude of the signal received to the average amplitude of background noise, also decreases when very thin slices are used.

The size of the FOV is another important consideration when using MRI to detect small erosions at the wrist and hand. A larger FOV allows the inclusion of more joints so that "capture" of damage progression is maximised, but spatial resolution can be compromised. In general, an FOV of $10 \mathrm{~cm}$ or less will allow greater spatial resolution and this is particularly the case for images obtained from high field $(1.5 \mathrm{~T})$ magnets with a surface coil. ${ }^{3}$ If a smaller FOV is used, however, this may mean that wrist and MCP joints cannot be imaged together. In one study of the rheumatoid hand,
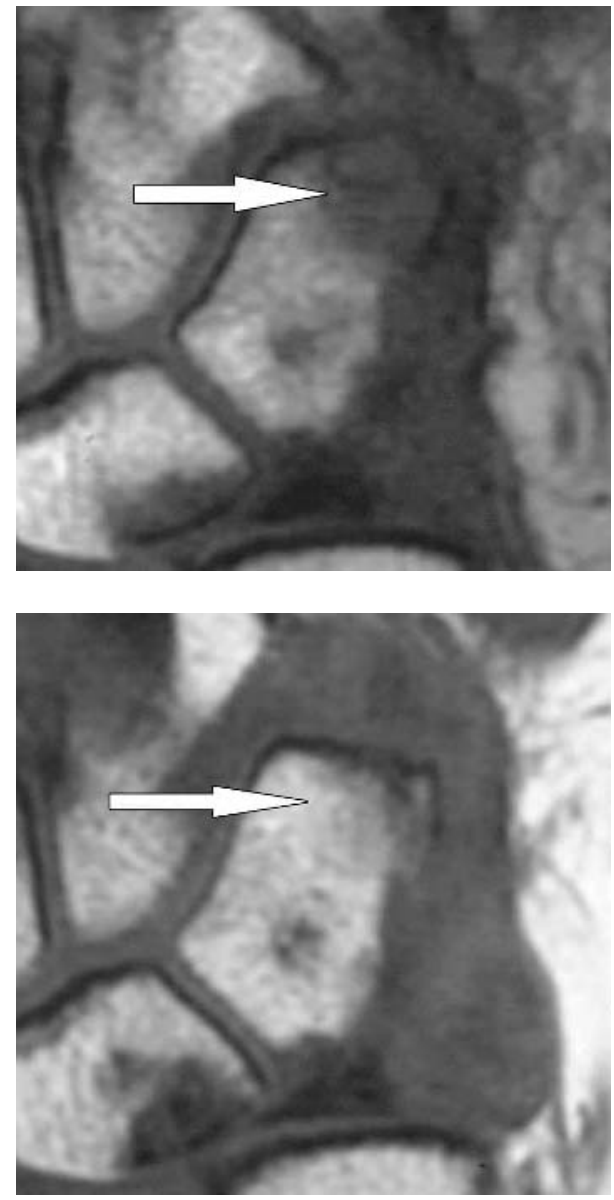

Figure 12 (A) $\mathrm{Tl}$ weighted coronal image of the wrist in a patient with early RA. Bone oedema is seen at the pole of the triquetrum with blurring of the dark cortical line, raising the possibility of an adjacent erosion. Bone oedema is also present more proximally within the triquetrum and also at the proximal border of the lunate. (B) $\mathrm{Tl}$ weighted coronal image one year later, showing that most of the bone oedema at the pole of the triquetrum has resolved. Bone oedema remains in the centre of that bone and is now more extensive in the lunate.

erosion scores were found to be similar when using a $10 \mathrm{~cm}$ and a $13 \mathrm{~cm}$ FOV but estimates of erosion volume were more variable when the larger FOV was utilised. ${ }^{8}$

\section{DISTORTION OF ANATOMY DUE TO DISEASE \\ Subluxation}

As is the case for plain radiography, imaging joints that have been damaged and altered anatomically by the disease process is challenging. Subluxation at MCP joints can make assessment of the degree of erosion at metacarpal heads more difficult. Similarly, gross deformity at the wrist causes confusion in identifying the individual carpal bones, especially if these assume new anatomical positions and have lost their characteristic shapes as in the example shown in fig 10.

\section{Irregular bony margins with adjacent synovitis}

At the wrist, many of the small carpal bones have irregular margins with indentations for ligamentous attachments. If there is florid synovitis, inflamed tissue with bright signal on $\mathrm{Tl}$ weighted post-gadolinium images, T2 weighted fat saturated or short tau inversion recovery (STIR) images will sit next to bone and can simulate pannus within a shallow erosion. ${ }^{9}$ Knowledge of the anatomy of the area is essential to avoid misinterpretation. 


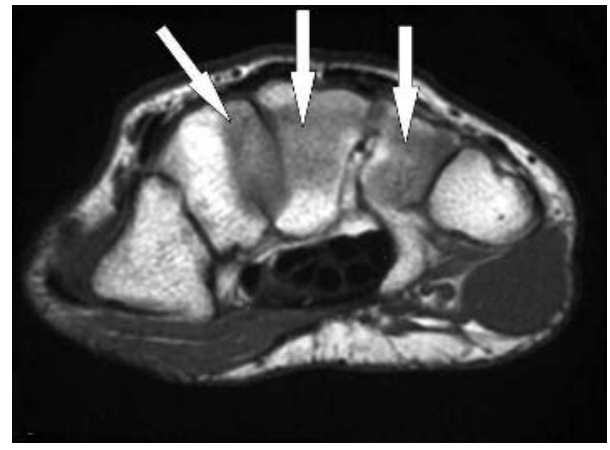

Figure $13 \mathrm{Tl}$ weighted axial image of the wrist showing reduced signal within the capitate, hamate, and trapezoid due to proximity of the bony border adjacent to the metacarpal bases.

\section{BONE EROSIONS AND BONE OEDEMA \\ Erosions versus bone oedema}

Erosions may be difficult to distinguish from focal regions of bone marrow oedema, especially on Tl weighted scans where both appear as regions of low signal. Bone oedema classically gives a bright signal on T2 weighted fat saturation, STIR and Tl weighted post-gadolinium images. However, erosions may also do so if they contain pannus. Erosions tend to have clearly defined margins and a clear cortical break, but sometimes there is bone oedema surrounding the erosion that makes distinction between the two lesions more difficult. ${ }^{10}$ Figure 11 shows an example from a patient with active RA of one year's duration, illustrating some of these difficulties. Figure 12 illustrates how bone oedema may be transient.
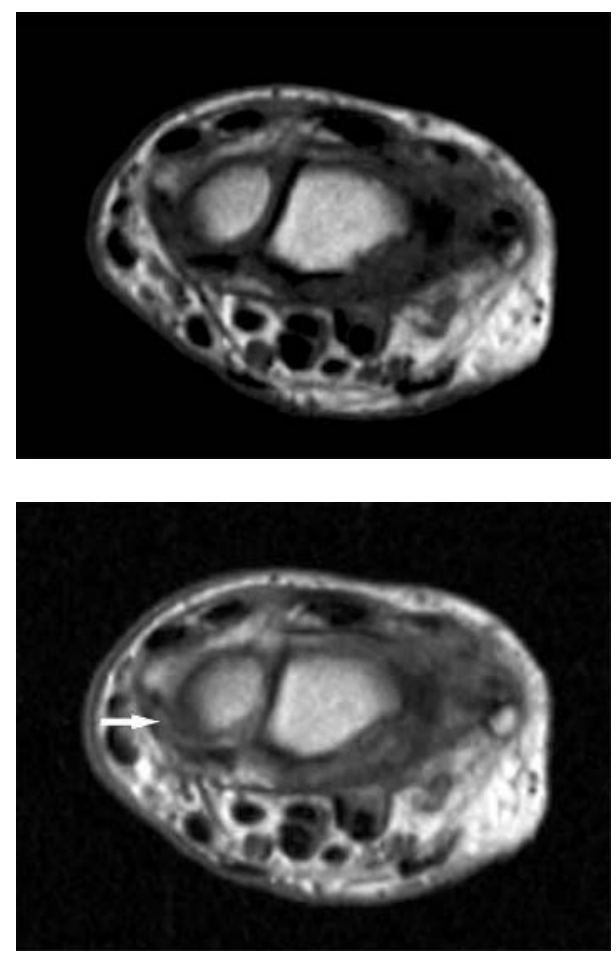

Figure 14 (A) T1 weighted axial image of the wrist from a normal control subject. (B) Postcontrast (gadolinium-DTPA) image of the same region revealing a thin rim of enhancing synovial membrane adjacent to the palmar aspect of the ulna (arrow).
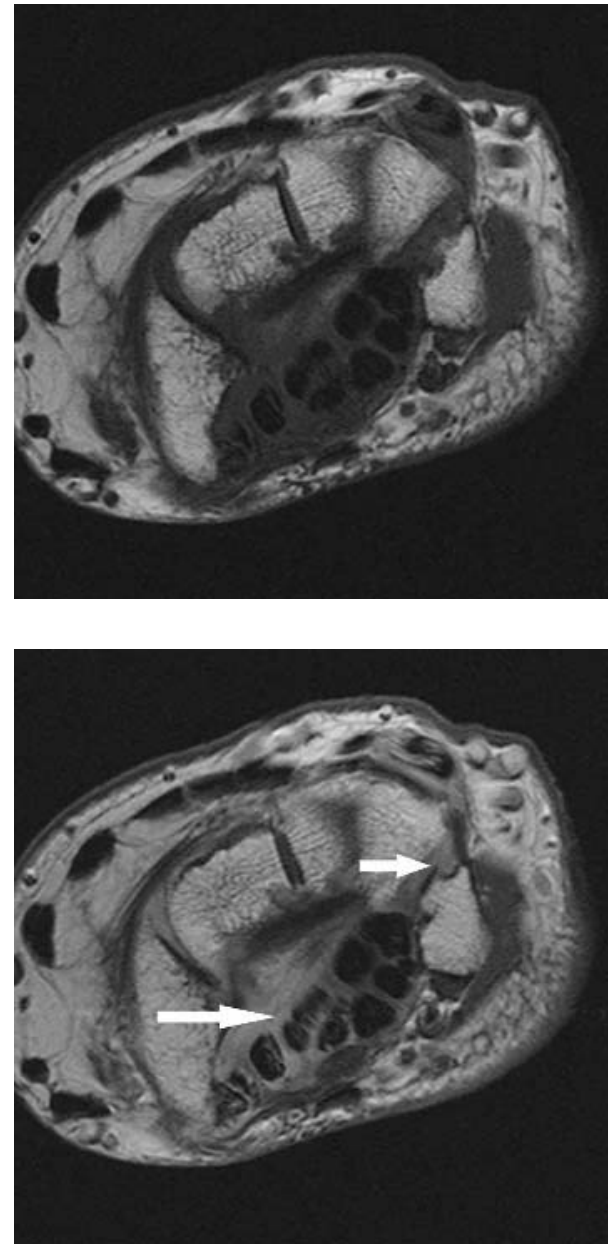

Figure 15 (A) $\mathrm{T} 1$ weighted axial image of the wrist. (B) Postcontrast T1 weighted axial image of the same region showing increased signal in the region of the flexor tendons (long arrow) plus low grade synovitis within the intercarpal joint (short arrow).

\section{Optimal sequences for visualising bone oedema}

On Tl weighted sequences, bone oedema appears as a low signal intensity region with poorly defined borders and a "feathery" appearance. ${ }^{11}$ For this appearance to show clearly, the $\mathrm{Tl}$ weighted sequence needs to have an adequately low echo time $(<15 \mathrm{~ms})$. Otherwise, the scan becomes "proton density" weighted and will not show the reduced signal of bone oedema. On T2 weighted fat saturation and STIR sequences, bone oedema appears as increased signal with an ill defined margin.

\section{Normal appearances mimicking bone oedema}

There is decreased signal on Tl weighted images where the edge of a bone approaches a joint, caused by partial volume artefacts from cortical bone and extraosseous tissues. This appearance can mimic bone oedema (fig 13) but other images taken perpendicular to the original will show normal signal.

\section{SYNOVITIS AND TENOSYNOVITIS}

\section{Synovial enhancement occurring in normal joints}

The thin layer of synovial membrane present in normal joints can enhance post gadolinium in some instances ${ }^{6}{ }^{12}$ (fig 14). For this reason, the definition of synovitis used in the RAMRIS system ${ }^{1}$ includes both the degree of postcontrast enhancement and the thickness of synovial membrane. 


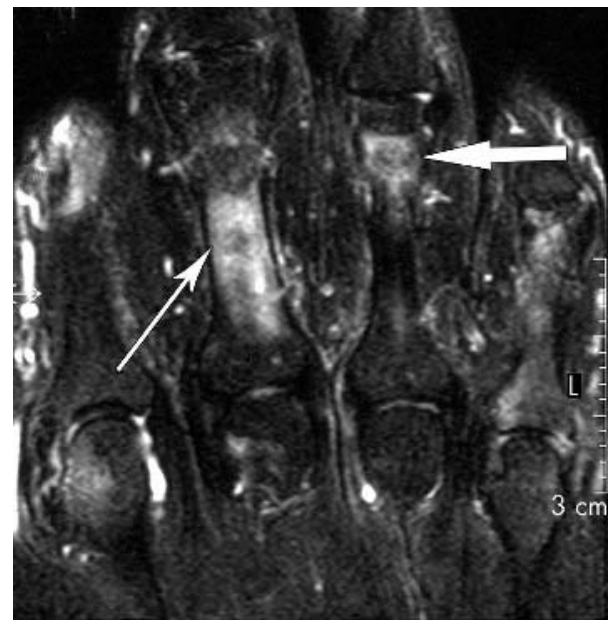

Figure 16 Short tau inversion recovery (STIR) coronal image of the fingers of a patient with rheumatoid arthritis covering the second to fifth metacarpophalangeal and proximal interphalangeal joints. Bright signal over the proximal phalanx of the third finger (thin arrow) resembles bone oedema but is actually due to inflammation within the flexor tendon sheath (tenosynovitis). True bone oedema is seen distally at the fourth proximal phalanx (wide arrow).

\section{Timing post gadolinium}

Following the injection of intravenous contrast (gadolinium) there is a time course for the development of enhancement within the synovial membrane dictated by the speed with which the contrast material moves through the circulation and small synovial vessels and then diffuses into the interstitium of the synovial membrane. If an area is scanned too soon after intravenous contrast has been given, enhancement will not be as great, potentially leading to underreading of the degree of synovitis. However, if the scan is performed too long after contrast injection, there is a slow fall-off of intensity, especially if there is an associated synovial effusion as the gadolinium contrast agent will equilibrate between the synovial membrane and the effusion. In that situation, the thickness of the synovial membrane may be overestimated if the space occupied by the effusion is mistakenly included. ${ }^{11}$ This could lead to error in quantifying synovitis.

\section{Tenosynovitis versus synovitis}

Knowledge of the complex anatomy of the wrist and hand is essential to avoid confusing synovitis with tenosynovitis. Both synovitis and tenosynovitis are characterised by increased signal on $\mathrm{Tl}$ weighted postcontrast and $\mathrm{T} 2$ weighted fat saturation images and are seen together on axial images of the wrist (fig 15).

\section{Tenosynovitis versus bone oedema}

On T2 weighted fat saturation scans there is potential for confusion between bright signal due to inflammation within tendon sheaths (tenosynovitis), as these run close to bone, and bright signal within the bone marrow itself due to bone oedema. An example is shown in fig 16.

\section{SUMMARY}

In this paper we have attempted to summarise some of the pitfalls which may be encountered when scoring rheumatoid joint inflammation and damage at the hand and wrist using the RAMRIS system. The nature of the imaging modality itself dictates that certain artefacts may occur, especially in regions where tissues of very different density are closely apposed. Usually, the experienced reader can interpret these appropriately using different sequences and views. Problems with movement and metal artefacts are fairly easily identified but may preclude scanning of the region in some instances. The normal anatomy of the hand is complex and structures such as interosseous ligaments and nutrient foramina need to be differentiated from erosions. The influence of slice thickness and FOV has been briefly alluded to as these will dictate image clarity and the ability to reliably identify small lesions. Applying the RAMRIS score to joints damaged by extensive erosion poses its own set of problems, especially at the wrist. The identification of erosions, bone oedema, synovitis and tenosynovitis has been described elsewhere in this atlas but these lesions can occasionally be mistaken for one another and frequently coexist in the rheumatoid hand. Thus, interpretation of MR images is challenging and requires knowledge of anatomy as well as an ability to recognise pathology. In addition, a working knowledge of the various MR sequences and how to apply these is essential to avoid the pitfalls mentioned above.

\section{ACKNOWLEDGEMENTS}

Drs N Stewart and A Doyle, Department of Radiology, Auckland City Hospital, New Zealand, and Dr A G Jurik, Department of Radiology, Aarhus University Hospital, Denmark, are acknowledged for their assistance in preparation of this manuscript.

The European League Against Rheumatism (EULAR) is acknowledged for financial support of the publication of this atlas.

\section{Authors' affiliations}

F McQueen, Department of Molecular Medicine and Pathology, Faculty of Medicine and Health Sciences, University of Auckland, Auckland, New Zealand

M Østergaard, Departments of Rheumatology, Copenhagen University Hospitals at Herlev and Hvidovre, Copenhagen, Denmark

C Peterfy, Synarc Inc, San Francisco, CA, USA

M Lassere, Department of Rheumatology, St George Hospital, University of NSW, Sydney, Australia

B Ejbjerg, Departments of Rheumatology, Radiology and MRI, Copenhagen University Hospital at Hvidovre, Copenhagen, Denmark P Bird, Department of Rheumatology, St George Hospital, University of NSW, Sydney, Australia

P O'Connor, Department of Radiology, Leeds General Infirmary, Leeds, UK

H Genant, Department of Radiology, University of California at San Francisco, San Francisco, CA, USA

R Shnier, Department of Diagnostic Imaging, Mayne Nickless, Sydney, Australia

P Emery, Academic Unit of Musculoskeletal Disease, University of Leeds, Leeds, UK

J Edmonds, Department of Rheumatology, St George Hospital, University of NSW, Sydney, Australia

P Conaghan, Academic Unit of Musculoskeletal Disease, University of Leeds, Leeds, UK

\section{REFERENCES}

1 Østergaard M, Peterfy C, Conaghan P, McQueen F, Bird P, Ejbjerg B, et al. OMERACT rheumatoid arthritis magnetic resonance imaging studies. Core set of MRI acquisitions, joint pathology definitions and the OMERACT RA-MRI scoring system. J Rheumatol 2003;30:1385-6.

2 New PF, Rosen BR, Brady TJ, Buonanno FS, Kistler JP, Burt CT, et al. Potential hazards and artifacts of ferromagnetic and non-ferromagnetic surgical and dental materials and devices in nuclear magnetic resonance imaging. Radiology 1983;147:137-48.

3 Bonel HM, Reiser M. Magnetic Resonance Imaging. In: Guglielmi G, van Kuijk C, Genant HK, eds. Fundamentals of hand and wrist imaging. Germany: Springer-Verlag, 2001:75-107.

4 Berquist TH, Morin RL. General technical considerations in musculoskeletal MRI. In: Berquist TH, ed. MRI of the musculoskeletal system, 3rd edn. Philadelphia: Lippincott-Raven Publishers, Mayo Clinic Foundation ${ }^{\odot}$, 1996:63-97.

5 Peterfy C, Linaris R, Steinbach L. Recent advances in magnetic resonance imaging of the musculoskeletal system. Radiol Clin North $\mathrm{Am}$ 1994; 32:291-311.

6 Ejbjerg B, Narvestad E, Rostrup E, Szkudlarek M, Jacobsen S, Thomsen HS, et al. Magnetic resonance imaging of wrist and finger joints in healthy subjects occasionally shows changes resembling erosions and synovitis as seen in rheumatoid arthritis. Arthritis Rheum 2004;50:1097-106. 
7 Bird $\mathbf{P}$, Lassere $M$, Shnier R, Edmonds J. What is the optimal slice thickness for assessment of erosive damage on MRI in the wrist and MCP joints of patients with rheumatoid arthritis? Ann Rheum Dis 2003;62(suppl 1):156

8 Bird P, Lassere M, Shnier R, Edmonds J. MR imaging and rheumatoid arthritis: What is the optimal field of view in wrist and MCP imaging? [Abstract] Ann Rheum Dis 2003;62(suppl 1):156-7.

9 McQueen FM. Magnetic resonance imaging in early inflammatory arthritis: what is its role? Rheumatology 2000;39:700-6.
10 Goldbach-Mansky R, Woodburn J, Yao L, Lipsky PE. Magnetic resonance imaging in the evaluation of bone damage in rheumatoid arthritis: a more precise image or just a more expensive one? Arthritis Rheum 2003;48:585-9.

11 Peterfy CG. Magnetic resonance imaging of rheumatoid arthritis: the evolution of clinical applications through clinical trials. Semin Arthritis Rheum $2001 ; 30: 375-96$.

12 Partik B, Rand T, Pretterklieber ML, Voracek M, Hoermann M, Helbich TH. Patterns of gadopentetate-enhanced MR imaging of radiocarpal joints of healthy subjects. AJR Am J Roentgenol 2002;179:193-7. 